\title{
CDK13 RNA Over-Editing Mediated by ADAR1 Associates with Poor Prognosis of Hepatocellular Carcinoma Patients
}

\author{
Xiuqing Donga Geng Chen ${ }^{a, c, d}$ Zhixiong Cai ${ }^{a, c, d}$ Zhenli Lia, Liman Qiu ${ }^{a, c}$ \\ Haipo Xu $u^{a, c}$ Yuan Yuan ${ }^{a, c}$ Xiaolong Liu ${ }^{a, c}$ Jingfeng Liu ${ }^{a, b, c}$
}

aThe United Innovation of Mengchao Hepatobiliary Technology Key Laboratory of Fujian Province, Mengchao Hepatobiliary Hospital of Fujian Medical University, Fuzhou, 'biver Disease Center, The First Affiliated Hospital of Fujian Medical University, Fuzhou, 'The Liver Center of Fujian Province, Fujian Medical University, Fuzhou, 'School of Life Science and Technology, Xi'an Jiaotong University, Xi'an, China.

\section{Key Words}

RNA-editing • Sequencing • Hepatocellular carcinoma • ADAR1 • Prognosis

\begin{abstract}
Background/Aims: Aberrant RNA editing, mediated by adenosine deaminases acting on RNA (ADAR), serves as a post-transcriptional event participating in tumorigenesis and prognosis. However, the RNA editing profiles during HCC progression and their clinical correlations remain unclear. Methods: Multiple tissue samples were collected from an advanced HCC patient. RNA-seq was performed to obtain the RNA editing profiles for each sample. Two RNA editing sites from CDK13 were further validated in $60 \mathrm{HCC}$ patients; and their potential regulatory mechanisms were investigated. Results: In-depth analysis of the RNA-seq data revealed a significant number of editing sites (632-816) in coding regions for each tissue sample, showing branched evolution during tumorigenesis and metastasis. Two editing sites (Q103R and K96R) in CDK13 showed significant over-editing in tumor, and these phenomenon were validated in $60 \mathrm{HCC}$ patients. Furthermore, the clinicopathological analysis revealed that these CDK13 over-editing sites were positively associated with TNM, PVTT and poor prognosis. In addition, the editing level of these sites were significantly correlated with the expression of ADAR1. Loss of function assays further proved that these CDK13 over-editing sites were mediated by ADAR1 in HCC cells. Conclusions: CDK13 RNA over-editing sites mediated by ADAR1 may serve as novel cancer driver events in HCC progression.
\end{abstract}

Xiuqing Dong, Geng Chen and Zhixiong Cai contributed equally to this work.

Xiao-Long Liu

and Jing-Feng Liu

KARGER
The United Innov. of Mengchao Hepatobiliary Technology Key Lab. of Fujian Prov., Mengchao Hepatobiliary Hospital of Fujian Med. Univ.; Xihong Road 312, Fuzhou 350025, Fujian, (China); Tel. +86 5918370 5927, E-Mail xiaoloong.liu@gmail.com / drjingfeng@126.com 


\section{Introduction}

Hepatocellular carcinoma (HCC) is one of the most common cancer with poor clinical outcomes, and it ranks the third leading cancer-related death in China [1]. HCC is associated with multiple high risk factors, including hepatitis B virus (HBV) or hepatitis C virus (HCV) infection, alcoholic cirrhosis and aflatoxin B1 (AFB1) contamination of food [2]. Similar as other solid tumors, the development of HCC also involves complicated genetic and epigenetic alterations. Due to the rapid development of next generation sequencing technology, recent studies have extensively focused on investigation of genomic DNA mutations and gene expression changes in HCC [3-7]. However, the mutations in genomic DNA are not the only driven force for HCC initiation and progression. Other events on RNA, such as RNA mutations, change of mRNA transcripts, also could significantly affect the initiation, development and prognosis of HCC.

RNA editing, a post-transcriptional event on RNA, was firstly discovered in trypanosome mitochondria [8]. In humans, the most popular type of RNA editing is A-to-I editing [9]. A-I editing is catalyzed by the enzyme family of adenosine deaminase acting on RNA (ADAR) , which can convert adenosine (A) to inosine (I) by deamination [10]. Subsequently, the splicing and translational machineries could recognize inosine as guanosine (G), therefore the result of A-I editing in coding regions may lead to amino acid substitution. Previous studies suggested that aberrant RNA editing events played a vital role in HCC development. For example, RNA over-editing of BLCAP which leads to a Tyr $\rightarrow$ Cys amino acid substitution, could stably promote cell proliferation by enhancing phosphorylation of AKT and further contribute to hepatocarcinogenesis [11]. Chen and his colleagues also suggested that increased ADAR1-mediated A-I editing of AZINI could promote cell proliferation and tumor progression through the neutralization of antizyme-mediated degradation of the target oncoproteins ornithine decarboxylase (ODC) and cyclin D1 (CCND1) in HCC[12]. In these studies, tumor over-editing sites are screened based on primary tumor and matched normal tissues, while profiling of RNA editing along with tumor recurrence and metastasis were rarely investigated and thus did not discriminate the trunk editing sites (commonly identified in all tumor sites) and recurrent/metastatic specific editing sites.

Here, we collected multiple tissue samples (including primary tumor, matched peritumor, recurrent tumors in segment VII and V of liver, portal vein tumor thrombus (PVTT) and metastatic lymph node) from a HBV related advanced HCC patient receiving two hepatectomy to analyze the RNA editing profiles during HCC progression. Our analysis discovered two RNA editing sites (Q103R and K96R) of CDK13 (cyclin-dependent kinase protein), which participated in pre-mRNA splicing and severed as a cancer driver gene in HCC. Then we explored the clinical significance and potential RNA editing mechanisms of CDK13 in large HCC cohorts. The results demonstrated that CDK13 RNA over-editing induced by ADAR1 were tightly associated with poor prognosis of HCC patients.

\section{Materials and Methods}

\section{Sample collection}

Six sequencing specimens were obtained from a HBV related advanced HCC patient who received surgical resection and other adjuvant therapies in Mengchao Hepatobiliary Hospital of Fujian Medical University over one 1-year clinical course. Primary HCC tissue and matched normal tissue were collected from the first surgery, and four recurrent/metastatic tumor tissues including the recurrent HCC in liver VII section (labeled as RT1), recurrent HCC in liver V section (labeled as RT2), portal vein tumor thrombus (labeled as MT1) and lymphatic node metastasis (labeled as MT2), were collected 5 months later at the second surgical resection (Fig. 1A). General deterioration then occurred at day 350 of follow-up. Treatment was stopped and the patient died 2 months later. Another 60 paired HCC tumor and matched peritumor samples were also obtained from Mengchao Hepatobiliary Hospital of Fujian Medical University. None of these patients received chemotherapy or other adjuvant therapies before surgical resection. The tissue samples were immediately frozen in liquid nitrogen after collection and kept until RNA extraction.

\section{KARGER}




\section{Cellular Physiology Cell Physiol Biochem 2018;47:2602-2612 \begin{tabular}{l|l} 
and Biochemistry Published 10.1159/000491656 & $\begin{array}{l}\text { D } 2018 \text { The Author(s). Published by S. Karger AG, Basel } \\
\text { www.karger.com/cpb }\end{array}$ \\
\hline
\end{tabular}}

Dong et al.: CDK13 RNA Over-Editing in HCC

All human sample collection procedures and usage of human samples were approved by the Institution Review Board of Mengchao Hepatobiliary Hospital of Fujian Medical University. The written consent was received from all of the participated patient in this study.

\section{Transcriptome Sequencing}

RNeasy Mini kits (Qiagen, Dusseldorf, Germany) were used to extract RNA from all samples according to the manufacturer's instructions. RNA purity was assessed by measuring its A260/A280 ratio; RNA Integrity Number (RIN) was assessed using the Bioptic Qsep100. After that, RNA samples were prcossed for the purification of poly-A containing mRNA molecules using oligo-dT beads. The obtained mRNAs were fragmented into small pieces, and copied into cDNA using reverse transcriptase and random primers. After the end repair process, the cDNA fragments were all appended with a single ' $\mathrm{A}$ ' base, and subsequent ligated with sequencing barcoded adapters. The ligated samples were enriched using PCR to construct the final cDNA library. Finally, transcriptome sequencing was performed on the Illumina HiSeq 3000 platform using paired end sequencing at Beijing Novogene Bioinformatics Technology Co, Ltd (Beijing, China).

\section{Calling of RNA editing sites}

RNA editing sites were identified using RNAEditor [13], which could identify RNA editing sites using RNA sequencing data alone. It mainly includes the following steps: (1) mapping reads to reference genome, (2) calling RNA variant, (3) filtering out known genomic SNPs, (4) identifying RNA editing sites. RNAEditor was downloaded from https: //rnaeditoruni-frankfurt.de. The implement of this tools was conducted according to its documentation. All parameters used were set to default as recommended. The annotation files were acquired from RNAEditor annotation bundles.

\section{Gene Ontology (GO) annotation and pathway enrichment analysis}

The genes corresponding to tumor specific RNA editing sites were extracted and subjected to The Database for Annotation, Visualization and Integrated Discovery (DAVID, http://david.abcc.ncifcrf. gov) for gene ontology and pathway enrichment analysis. The enrichment results were annotated using KOBAS3.0[14].

\section{Verification of RNA editing sites}

RNA samples were prepared using RNeasy Mini kits (Qiagen, Dusseldorf, Germany). Template cDNA synthesis was done on $2 \mu \mathrm{g}$ of total RNA using Transcriptor First strand cDNA Synthesis Kit (Roche, Basel, Switzerland) with random primers. RNA editing sites was verified using RT-PCR. The PCR primers were listed as follows: CDK13, p.K96R, forward primer: 5'-CTCTGGAGGTCAAGCGGC-3',reverse primer: 5'-GACTGGGAGCTCACATCCTC-3'; p.Q103R, forward primer: 5'-CTCTGGAGGTCAAGCGGC-3', reverse primer: 5'-GACTGGGAGCTATCCTC-3'. The PCR product was subjected to Sanger sequencing, and the editing levels were calculated with ab1 Peak Reporter (https://apps.thermofisher.com/ab1peakreporter) based on the peak-height ratio of 5 . The over-editing events were defined by an increase of more than $20 \%$ editing in tumor samples compared with match normal samples [12].

\section{Determination of ADAR1 expression}

Real-time PCR was employed to quantify ADAR1 expression in HCC tissues and matched adjacent tissues. cDNA was prepared as described above, and Real-time PCR was performed on ABI 7500 Realtime PCR System (applied Biosystems) with SYBR green qPCR Kit (DBI Bioscience, Ludwigshafen, Germany) by following primers: ADAR1, forward primer: 5'-CCCTTCAGCCACATCCTTC-3', reverse primer: 5'-GCCATCTGCTTTGCCACTT-3'; GAPDH, forward primer: 5'-GGGAAACTGTGGCGTGAT-3', reverse primer: 5'GAGTGGGTGTCGCTGTTGA-3'. Expression level of GAPDH was used as internal control. The relative target gene expression in HCC tissues and matched adjacent tissues were calculated by $\triangle \mathrm{CT}$ method.

\section{Cell culture}

Human hepatoma cell line Bel-7402 was obtained from Shanghai Cell Bank of Chinese Academy of Sciences. Bel-7402 cells were maintained in RPMI-1640 culture medium (Gibco, Grand Island, NY, USA) supplemented with $10 \%$ fetal bovine serum (Gibco) and $100 \mathrm{U} / \mathrm{mL}$ penicillin and $100 \mathrm{ug} / \mathrm{ml}$ streptomycin (Gibco), and cultured at $37^{\circ} \mathrm{C}$ with $5 \% \mathrm{CO} 2$. 


\section{RNA interference}

siRNA sequences targeting the ADAR1 gene were designed and synthesized by RiboBio Co. Ltd, Guangzhou, China. The sequences were designed as follow: siADAR1 (5'-CGCAGAGUUCCUCACCUGUATT-3'). Negative control siNC was provided by RiboBio Co. Then, the siRNAs were transfected into Bel-7402 cells in 6-well plates using Lipofectamine 3000 (Life Technologies, Grand Island, NY, USA) reagent in accordance with the manufacturer's instructions. 6 hours after transfection, the culture medium was replaced by fresh RPMI-1640 (Gibco) culture medium. Then, the transfected cells were incubated for another 48 hours and subsequently collected for analysis.

\section{Western Blot}

Bel-7402 cells were lysed using RIPA buffer (0.5 M Tris- $\mathrm{HCl}, \mathrm{pH} 7.4,1.5 \mathrm{M} \mathrm{NaCl}, 2.5 \%$ deoxycholic acid, 10\% NP-40, 10 mM EDTA) containing protease inhibitor cocktail (Roche, Basel, Switzerland).Total protein extracts were subjected to protein gel electrophoresis using 10\% SDS-PAGE, and transferred to nitrocellulose membranes(Life Technologies, Grand Island, NY, USA). After blocking with PBS containing 5\% BSA, the membranes were incubated with primary ADAR1 antibody (1:1000 dilution; ab88574; Abcam, Cambridge Science Park, UK) and $\beta$-actin antibody (1:5000 dilution; Transgen, Beijing, China) at $4{ }^{\circ} \mathrm{C}$ overnight, followed by incubation with second HRP conjugated antibody (1:5000 dilution; Transgen) at room temperature for $1 \mathrm{~h}$. The protein express level was visualized by enhance chemiluminescence.

\section{Statistical analysis}

The SPSS statistical software package (version 19.0, SPSS, Chicago, IL, USA) was used to perform the data analysis. The editing level of $C D K 13$ in tumor samples and match normal samples were compared using paired Wilcoxon test. Categorical data between $C D K 13$ editing level and the clinicopathological features of patients were analyzed by Fisher's exact test. Kaplan-Meier plots and log-rank tests were performed for overall survival analysis. Comparison of $A D A R 1$ and $A D A R 2$ expression in tumor and matched peritumor samples were conducted using one sample t-test. ADAR1 levels in any two groups of clinical samples were tested with a two-tailed unpaired Student's t-test. $p<0.05$ was considered as statistically significant.

\section{Results}

\section{Calling of RNA editing sites for tumor samples from an advanced HCC patient}

To analyze the RNA editing profiles during HCC progression, a HBV related advanced HCC patient sequentially receiving two hepatectomy were enrolled. After suffering 15 years of decompensate hepatitis-B-related cirrhosis, this patient was first diagnosed with primary HCC by routine MR scan and further received surgical operation. After 5 months, the recurrence happened with multiple intrahepatic metastases, and then he received surgical debulking. During his two hepatectomy, multiple tissue samples (NT1, PT1, RT1, RT2, MT1and MT2) were collected for sequencing analysis. RNA sequencing was performed for all tissue samples, and obtained more than 82.36 million sequence reads for each sample. Bioinformatic tool "RNAEditor" was applied to analyze RNA editing in both tumor and matched peritumor samples. As shown in Fig. $1 \mathrm{~B}$, the results showed that an average of 203, 992 potential editing sites (ranging from 111, 689 to 280,613) were detected in these 6 tissue samples; noteworthily, HCC samples shown an average 222453 of A to I RNA editing events- nearly twice as much as in normal tissue samples (111689). Consistent with previous studies, the majority of RNA editing sites were discovered in Alu region $[15,16]$. Moreover, only $16.6 \%$ of these editing sites have been identified previously in DARNED [17], demonstrating that we were possibly identified high proportion of new RNA editing sites in this HCC patient. Visualization with Circos showed that these editing sites were evenly distributed on each chromosome (Fig. 1C). In addition, by examining the sequence near our detected editing sites, we interestingly discovered that the $\mathrm{G}$ base in the nearby upstream of editing site was decreased while in the nearby downstream of editing site was increased, which were well consistent with the reported sequence preference of ADARs targeting [18] (Fig. 1D). 
RNA editing profiles during HCC progression

RNA editing events occurring within coding regions may cause amino acid substitutions in translation process and further affect protein conformation and interaction. Based on the site location, these RNA editing sites could be divided into 9 categories: 3'-UTR, 5'-UTR, upstream, splicing, ncRNA, intronic, intergenic, exonic and downstream regions. As shown in Fig. 2A and 2B among above mentioned 6 tissue samples, averagely $54.2 \%$ of RNA editing sites were identified in intronic region, $2.82 \%$ in downstream region, $27.7 \%$ in intergenic region, $8.44 \%$ in ncRNA region, $0.03 \%$ splicing region, $0.91 \%$ in upstream region, $5.39 \%$ in $3^{\prime}$-UTR region, $0.14 \%$ in $5^{\prime}$ UTR region; while only $0.39 \%$ (3299) editing sites were located in the coding region (632 in NT1, 732 in PT1, 797 in RT1, 816 in RT2, 749 in MT1, 797 in MT2). After filtering RNA editing acquired in peritumor sample (NT1), we obtained an average of 498 tumor specific RNA editing sites (range, 459-531); and only 95 of these RNA editing sites were shared between primary tumor and recurrent/metastatic tumors, indicating high heterogeneity of RNA editing events among tumor lesions (Fig. 2C). For better demonstration of the heterogeneity across tissue samples, phylogenetic trees were constructed based on RNA editing site distribution of each sample (Fig. 2D). Among these identified sites, 228 editing sites were common among all tissue samples, 95 of them were shared in all cancer tissues, 11 were specifically related to recurrences/metastases and others were unclassified or private since they did not follow any clear distribution pattern. Consistent with previous results in DNA mutations, this patient showed clear evidence of branched evolution during tumorigenesis and metastasis [19].

To further screen out the functional editing sites, 46 nonsynonymous sites from 12 genes were extracted from 95 tumor specific RNA editing sites that commonly identified in all tumor samples (Fig. 2E). Then Gene Ontology (GO) annotation and pathway enrichment analysis were performed on these 12 genes to determine the significantly affected biological processes. Enrichment in nuclear division, positive regulation of transmembrane transport and organelle fission pathways were observed in GO annotation (Fig. 2F). Meanwhile, pathway enrichment analysis revealed that several glycosylation associated pathways were significantly enriched; interestingly, transcriptional regulation by TP53 pathway, which is crucial for the formation of most tumors, were also observed (Fig. 2G). By deciphering detailed genes in pathways, cyclin-dependent kinase protein kinase 13 (CDK13), which has been report to play a vital role in HCC development, was specifically assigned to transcriptional regulation by TP53 pathway. Based on above results, CDK13 was selected as the candidate for further large cohort study.

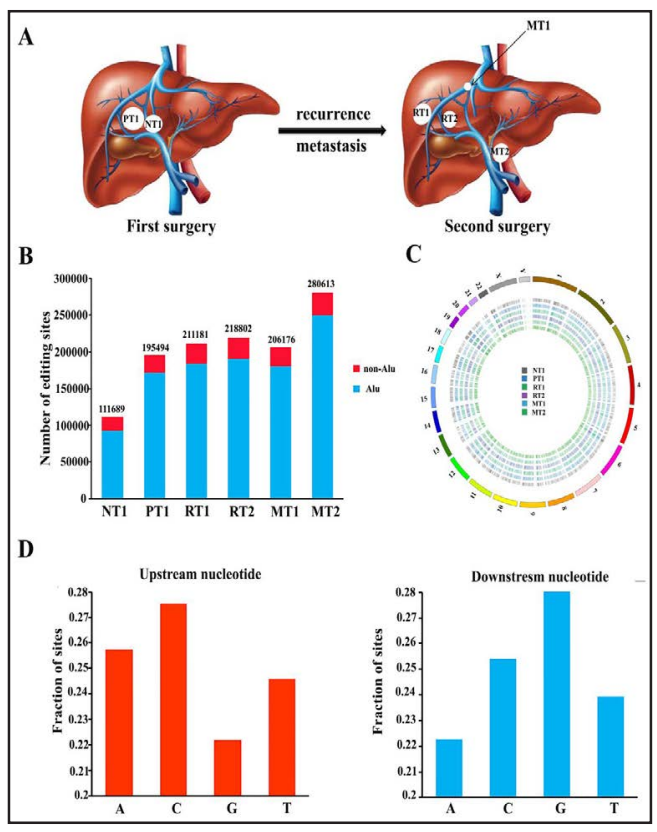

Fig. 1. Global identification of RNA editing sites in six tissues collected from an advanced NT1 (normal liver sample), PT1 (primary liver RT2 (Recurrent tumor in liver VII section), MT1 (portal vein tumor thrombus), MT2(lymphatic node Metastasis). (B) The number of RNA editing sites identified in six samples, including in Alu and non-Alu regions. (C) Distribution of (shown by exterior circle) in six samples. (D) The fraction of each nucleotide type in nearby upstream and downstream of the editing site. the patient during two surgeries, labelled as 
Identification of CDK13 RNA over-editing events in HCC

CDK13, a member of the cyclin-dependent serine/threonine protein kinase family, participated in pre-mRNA splicing and gene transcription in HCC. In this study, we identified two tumor specific editing sites in CDK13: the substitution of glutamine with arginine at residue 103 (Q103R) and the substitution of Lysine with arginine at residue 96 (K96R). To confirm these editing events of CDK13 in above RNA-sequencing samples, we perform sanger sequencing for both gDNA and cDNA. As shown in Fig. 3A and 3B, high frequency of RNA mutation in CDK13 Q103R and K96R site were detected in all five tumor samples (without in matched peritumor), but not detected corresponding mutation in DNA sequence. To further assess these two RNA editing events of CDK13 in HCC, another 60 paired HCC tumor samples and matched peritumor samples were enrolled for analysis. As shown in Fig 3C and 3D, these HCC samples had significant higher editing levels of CDK13 Q103R and K96R sites when compared with matched peritumor samples $(p<0.001)$. Approximately $43 \%$ $(26 / 60)$ of HCC patients had CDK13 over-editing at Q103R site and $60 \%(36 / 60)$ at K96R site; in total, $65 \%(39 / 60)$ of HCC patients had CDK13 over-editing events (Q103R or K96R). These results suggested that Q103R and K96R of CDK13 may participate in HCC tumorigenesis.

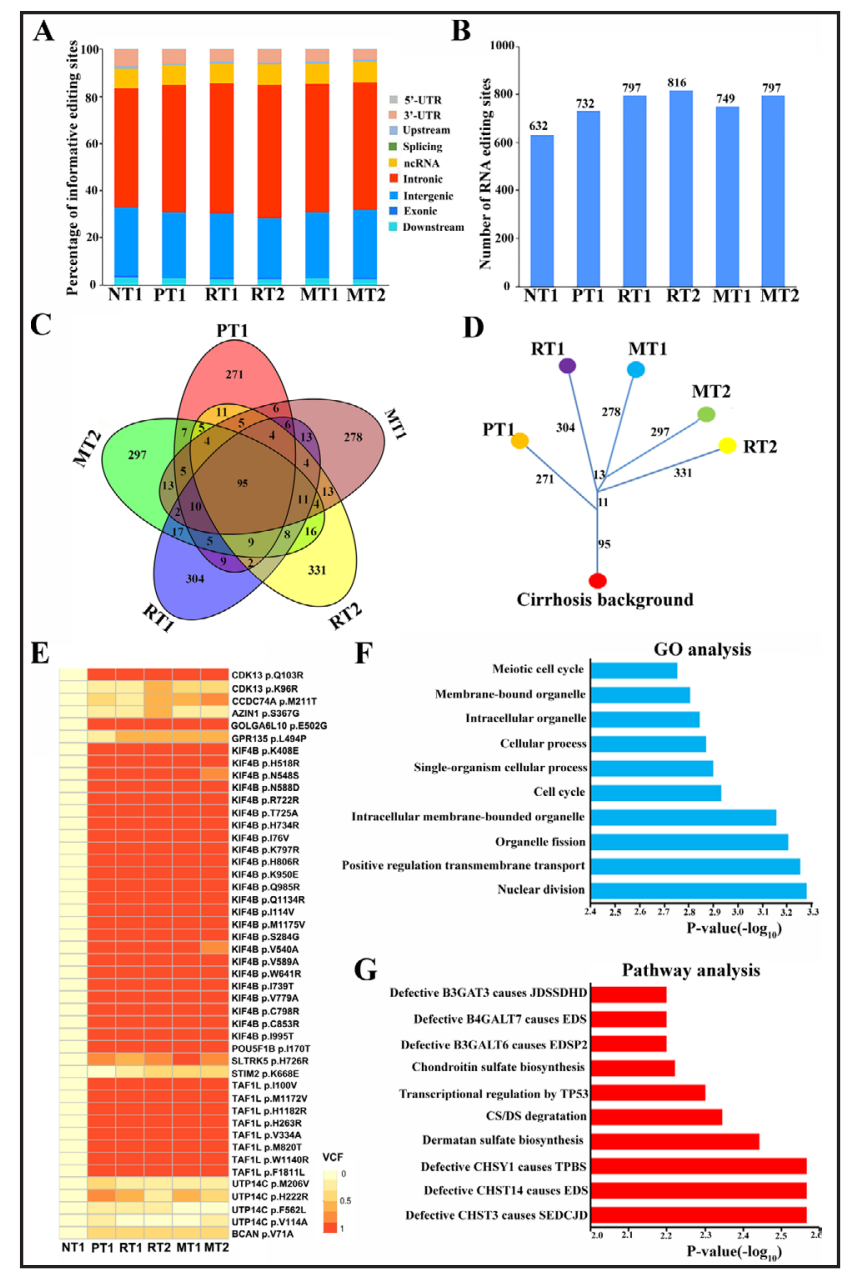

Fig. 2. The profiles of RNA editing sites in six samples collected from an advanced HCC patient. (A) The distribution of editing sites in each functional category. (B) The number of editing sites in coding regions. (C) Venn diagram of the number of tumor-specific editing sites in coding regions. (D) Tumor phylogenetic tree inferred by distribution of RNA editing sites in tissue samples; each branch length correlates with the number of editing sites on the branch as indicated. (E) The profiles of 46 non-synonymous tumor-specific RNA editing sites in coding regions. (F, G) Gene Ontology (GO) annotation and pathway enrichment analysis of the 12 tumorspecific genes. The $\mathrm{X}$-axis shows the enrichment significance presented with - $\log 10$ (P-value).

\section{CDK13 over-editing positively}

associates with poor prognosis of HCC

To investigate the role of CDK13 over-editing in HCC progression, we examined the association between the levels of these two editing sites and the clinical characteristics of above 60 HCC patients. As summarized in Table 1, CDK13 over-editing at Q103R site in tumors was statistically correlated with the presence of live cirrhosis $(p=0.031)$, TNM stage $(p=0.044)$ and presence of PVTT $(p=0.031)$, while K96R site was significantly correlated with TNM stage $(p=0.002)$ and the presence of PVTT ( $p=0.023)$. Furthermore, Kaplan-Meier analysis showed that the HCC patients with RNA over-editing of CDK13 at Q103R site or K96R site had a significant shorter OS time ( $p=0.048$ and $p=0.045$ respectively, Fig. $4 \mathrm{~A}$ and 4B). To

\section{KARGER}


improve the prognostic value of those RNA over-editing sites in HCC patients, AFP level was enrolled for further evaluation. As shown in Fig. 4C, the patients with $C D K 13$ over-editing ( + ) and AFP (+) had significant shorter overall survival than those with CDK13 over-editing (+) and AFP (-) or CDK13 over-editing $(-)$ and AFP (+), especially for those with $C D K 13$ over-editing $(-)$ and AFP ( -$)(p=0.002)$. These data suggested that RNA overediting of CDK13 at Q103R or K96R might represent a novel potential prognosis biomarker for HCC.

\section{Potential mechanism of CDK13 RNA editing identified in HCC \\ RNA editing is catalyzed by} ADAR family, which includes three members: ADAR1, ADAR2 and ADAR3. Among them, ADAR1 and ADAR2 are expressed in most tissues, whereas ADAR3's expression is limited to the brain $[20,21]$. To clarify potential mechanism corresponding to CDK13 RNA editing in HCC, we analyzed the correlations of ADAR expression with the editing level of $C D K 13$ Q103R and K96R sites. Firstly, we compared the expression levels of $A D A R 1$ and $A D A R 2$ in the six sequenced samples. The results showed that the expression of ADAR1 was significantly increased in tumor samples compared with matched peritumor samples, especially in recurrence/ metastasis tissues, while ADAR2 only showed slight increase in tumor samples $(p=0.001$ and $p=0.10$, respectively, Fig. $5 \mathrm{~A}$ and 5B). The increased ADAR enzyme expression also explained the phenomenon that RNA editing events were increased in tumor samples.

The Scatter plot showed that editing levels of CDK13 Q103R and K96R sites were positively correlated with $A D A R 1$ expression ( $p=0.012$ and $p=0.006$, respectively, Fig. 5C

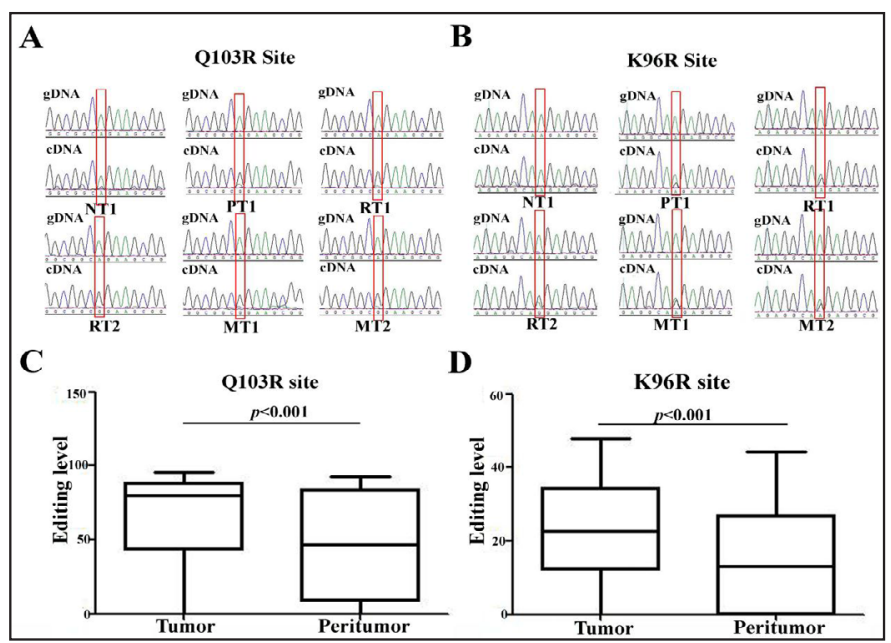

Fig. 3. Identification of CDK13 RNA over-editing events in HCC samples. (A, B) Sanger sequencing of both gDNA and cDNA of CDK13 Q103R site and K96R site in six tissue samples collected from an advanced HCC patient, respectively. Green indicates wild A base, Black indicates edited G base. (C) The RNA editing level of CDK13 Q103R site in large HCC cohort. (D) The RNA editing level of CDK13 K96R site in large HCC cohort.

Table 1. Associations between the editing levels of CDK13 Q103R and K96R sites and clinicopathological characteristics of 60 hepatocellular carcinoma patients. ${ }^{*} \mathrm{p}<0.05$; ${ }^{* *} \mathrm{p}<0.01$; oe: overediting

\begin{tabular}{|c|c|c|c|c|c|c|}
\hline \multirow{2}{*}{$\begin{array}{l}\text { Clinical } \\
\text { Characteristics }\end{array}$} & \multicolumn{3}{|c|}{ Q103R site } & \multirow[b]{2}{*}{ oe $(-)$} & \multicolumn{2}{|c|}{ K96R site } \\
\hline & oe $(-)$ & oe $(+)$ & $\mathrm{P}$-value & & $\mathrm{oe}(+)$ & P-value \\
\hline \multicolumn{7}{|l|}{ Sex } \\
\hline Male & 32 & 23 & 0.64 & 23 & 32 & 0.64 \\
\hline Female & 2 & 3 & & 1 & 4 & \\
\hline \multicolumn{7}{|l|}{ Age, years } \\
\hline$<55$ & 20 & 16 & 0.83 & 14 & 22 & 0.83 \\
\hline$\geq 55$ & 14 & 10 & & 10 & 14 & \\
\hline \multicolumn{7}{|l|}{ AFP, ng / mL } \\
\hline$<400$ & 23 & 15 & 0.43 & 17 & 21 & 0.33 \\
\hline$\geq 400$ & 11 & 11 & & 7 & 15 & \\
\hline \multicolumn{7}{|l|}{ HBV DNA } \\
\hline$<500$ & 13 & 12 & 0.54 & 10 & 15 & 1 \\
\hline$\geq 500$ & 21 & 14 & & 14 & 21 & \\
\hline \multicolumn{7}{|l|}{ Cirrhosis } \\
\hline Absent & 6 & 0 & $0.031^{*}$ & 2 & 4 & 1 \\
\hline Present & 28 & 26 & & 22 & 32 & \\
\hline \multicolumn{7}{|l|}{ Tumor number } \\
\hline Single & 30 & 21 & 0.48 & 22 & 29 & 0.29 \\
\hline Multiple & 4 & 5 & & 2 & 7 & \\
\hline \multicolumn{7}{|l|}{ Tumor size, $\mathrm{cm}$} \\
\hline$<5$ & 19 & 15 & 0.9 & 17 & 17 & 0.07 \\
\hline$\geq 5$ & 15 & 11 & & 7 & 19 & \\
\hline \multicolumn{7}{|l|}{ TNM stage } \\
\hline I-II & 31 & 18 & $0.044^{*}$ & 24 & 25 & $0.002 * *$ \\
\hline III-IV & 3 & 8 & & 0 & 11 & \\
\hline \multicolumn{7}{|l|}{ PVTT } \\
\hline Absent & 25 & 12 & $0.031^{*}$ & 19 & 18 & $0.023^{*}$ \\
\hline Present & 9 & 14 & & 5 & 18 & \\
\hline \multicolumn{7}{|c|}{ Tumor differentiation } \\
\hline I-II & 12 & 6 & 0.306 & 7 & 11 & 0.9 \\
\hline III-IV & 22 & 20 & & 17 & 25 & \\
\hline
\end{tabular}
KARGER 


\section{Cellular Physiology and Biochemistry}

Cell Physiol Biochem 2018;47:2602-2612

\begin{tabular}{l|l}
\hline DOI: $10.1159 / 000491656$ & (c) 2018 The Author(s). Published by S. Karger AG, Basel
\end{tabular}

Dong et al.: CDK13 RNA Over-Editing in HCC and 5D), but not significantly associated with the expression of ADAR2 $\quad(p=0.126$ and $p=0.19$, respectively, Fig. $5 \mathrm{E}$ and $5 \mathrm{~F}$ ). To further confirm the relationship between the level of CDK13 RNA editing and the expression of $A D A R 1$, we evaluated the ADAR1 expression in above 60 paired HCC samples by using realtime PCR. Then these patients were divided into two groups with low level and high level in ADAR1 based on its median expression level in all 60 tumor samples. As shown in Fig. $6 \mathrm{~A}$ and $6 \mathrm{~B}$, the editing level of CDK13 Q103R site and K96R were significantly associated with the expression of ADAR1 $(p=0.01$ and $p=0.003$, respectively ). These results suggested that $C D K 13$ editing might be mainly mediated by ADAR1.

ADAR1 is responsible for CDK13 RNA editing in HCC

To further examine whether abnormal expression of ADAR1 could affect the editing level of $C D K 13$, the HCC cell line Bel-7402 with high expression of ADAR1 was used for knockdown experiment. As shown in Fig. 6C, ADAR1 was successfully knocked down in Bel7402 cells. Then, we evaluated the levels of those two CDK13 editing sites by using sanger sequencing. As shown in Fig. 6D, knockdown of ADAR1 in Bel7402 cells could significantly decrease the editing level of $C D K 13$ Q103R site (41\%). Unfortunately, due to the low frequency of RNA editing in CDK13 Q96R in HCC cell lines $(<5 \%)$, we cannot accurately detect the editing level change in Bel-7402 cells after knocked down of ADAR1 expression（data not shown）. Hence, the potential regulator of this editing site still need to be further verified in HCC cells. These results suggested that ADAR1 is responsible for CDK13 RNA editing in HCC.

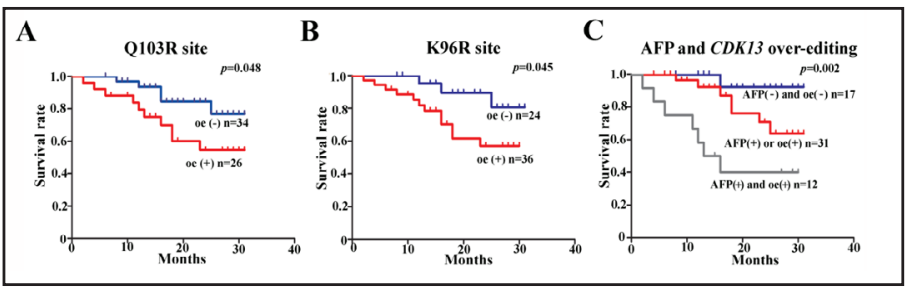

Fig. 4. Kaplan-Meier analysis of the association between CDK13 RNA-editing and HCC patients' overall survival. (A, B) CDK13 Q103R site or K96R site over-editing was significantly correlated with short overall survival time in HCC patients. (C) Comparison of the overall survival of three groups stratified by AFP level and CDK13 overediting level. oe: over-editing.

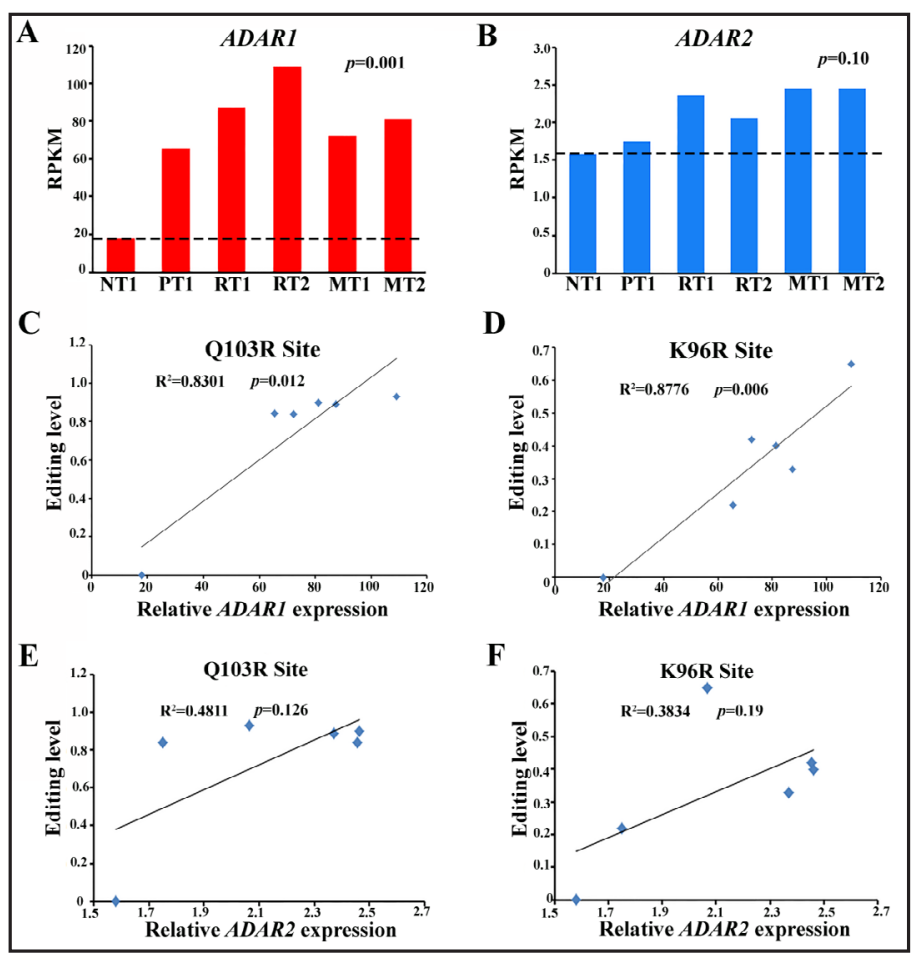

Fig. 5. The correlation between the RNA editing level and the expression of ADAR1, ADAR2. (A) Comparison of ADAR1 expression in six sequenced samples collected from an advanced HCC patient. (B) Comparison of ADAR2 expression in six sequenced samples collected from an advanced HCC patients. (C) The relationships between ADAR1 expression and the RNA editing level of CDK13 Q103R site. (D) The relationships between ADAR1 expression and RNA editing level of CDK13 K96R site. (E) The relationships between ADAR2 expression and the RNA editing level of CDK13 Q103R site. (F) The relationships between ADAR2 expression and RNA editing level of CDK13 K96R site. 
Discussion

RNA editing is defined as a post-transcriptional event that modifies RNA nucleotides from their complementary genomic sequence. Deciphering tumor special RNA editing distribution in HCC, especially profiling of RNA editing along with tumor progression, could provide a novel angle for understanding HCC progression. In our study, we investigated the spatial and temporal distribution of RNA editing events during the development of HCC by analyzing multiple tissue samples collected from an advanced HCC patient. Our analysis revealed that high heterogeneity of RNA editing also existed, which was a common phenomenon in DNA level. These data might provide deep insights of how RNA editing events varied during tumorigenesis and metastasis.

CDK13 is a member of the cyclin-dependent serine/threonine protein kinase family, with a conserved central CTD kinase domain in C-terminal region and a degenerate arginine/serine-rich (RS) domain in N-terminal region. They are involved in gene transcription and Pre-mRNA Splicing [2224]. Recent studies have shown that aberrant levels of $C D K 13$ were associated with several cancers. It was frequently up-regulated in HCC and colorectal cancer, suggesting CDK13 has an important influence on tumor development and progression [25]. Besides the overexpression of $C D K 13$, RNA editing of $C D K 13$ also played an important role in cancer development such as in glioblastoma cells [26]. However, the role of CDK13 over-editing in hepatocarcinogenesis and the potential regulatory mechanism are rarely studied in above literatures. In this study, we discovered two over-editing sites (Q103R and K96R) in the N-terminal region of CDK13, whose conformation is crucial for interacting with p32 (a regulating partner of the ASF/ SF2 splicing factor), were specifically detected in all tumor samples from the advanced HCC patient. Q103R site which has been discovered in human brain and in A172 glioblastoma cells $[26,27]$, while the other site (K96R) has never been reported before. Clinicopathological analysis showed that these two CDK13 over-editing sites were closely associated with the malignant process and prognosis of HCC, and may act as an "driver mutation" on RNA level participating in the development of HCC.

ADAR1, which is responsible for binding to double stranded RNA (dsRNA), could catalyze A to I editing by deamination [28]. Recent studies have shown that overexpression of ADAR1 could result in an increased A to I editing and further exert great influence in gene translation in HCC $[12,29,30]$. Consistently in this study, we also discovered that the overexpression of ADAR1 transcripts in HCC associated with the increased number of RNA editing events, suggesting that ADAR1 played crucial roles in HCC post-transcriptional regulation. Furthermore, the results also demonstrated that CDK13 editing pattern was significantly affected by the expression level of $A D A R 1$, indicating that $A D A R 1$ might serve as an "regulator" to participate in CDK13 over-editing in HCC progression. 


\begin{tabular}{|c|c|c|}
\hline \multirow[t]{2}{*}{ Cell } & \multicolumn{2}{|c|}{ Cell Physiol Biochem 2018;47:2602-2612 } \\
\hline & $\begin{array}{l}\text { DOI: } 10.1159 / 000491656 \\
\text { Published online: July } 11,2018\end{array}$ & $\begin{array}{l}\text { O } 2018 \text { The Author(s). Published by S. Karger AG, Basel } \\
\text { www.karger.com/cpp }\end{array}$ \\
\hline
\end{tabular}

\section{Conclusion}

In summary, as the first detailed analysis of RNA editing profiles along with HCC progression, our study provides new understanding of tumor heterogeneity in RNA levels. Moreover, we also discovered that two over-editing sites (Q103R and K96R) of CDK13, which were specifically modified by ADAR1, were more abundant in HCC tumor tissues and closely associated with poor prognosis of HCC patients. Accordingly, CDK13 over-editing may serve as a novel driver event in the pathogenesis of HCC.

\section{Acknowledgements}

This work was supported by the Science and Technology major project of Fuzhou (Grant No. RongKe 16 in 2016); the Natural Science Foundation of Fujian Province (Grant No.2018J01145); the Scientific Foundation of Fuzhou City (Grant No. 2016-S-124-11 and Grant No. 2017-S-131-3 ); the Startup Fund for scientific research, Fujian Medical University (Grant No. 2016QH083 and Grant No.2016QH085); the Scientific Foundation of Fuzhou Health Department (Grant No. 2017-S-wq38); the Science and Technology development project of central government guiding local government(Grant No.2017L3017); Joint Funds of Fujian Provincial Health and Education Research (WJK2016-2-25).

\section{Disclosure Statement}

No conflict of interests declared.

\section{References}

-1 Chen W, Zheng R, Baade PD, Zhang S, Zeng H, Bray F, Jemal A, Yu XQ He J: Cancer statistics in China, 2015. CA Cancer J Clin 2016;66:115-132.

$\rightarrow 2$ El-Serag HB, Rudolph KL: Hepatocellular carcinoma: epidemiology and molecular carcinogenesis. Gastroenterology 2007;132:2557-2576.

- 3 Schulze K, Imbeaud S, Letouze E, Alexandrov LB, Calderaro J, Rebouissou S, Couchy G, Meiller C, Shinde J, Soysouvanh F, Calatayud AL, Pinyol R, Pelletier L, Balabaud C, Laurent A, Blanc JF, Mazzaferro V, Calvo F, Villanueva A, Nault JC, Bioulac-Sage P, Stratton MR, Llovet JM, Zucman-Rossi J: Exome sequencing of hepatocellular carcinomas identifies new mutational signatures and potential therapeutic targets. Nat Genet 2015;47:505-511.

4 Fujimoto A, Totoki Y, Abe T, Boroevich KA, Hosoda F, Nguyen HH, Aoki M, Hosono N, Kubo M, Miya F, Arai Y, Takahashi H, Shirakihara T, Nagasaki M, Shibuya T, Nakano K, Watanabe-Makino K, Tanaka H, Nakamura H, Kusuda J, Ojima H, Shimada K, Okusaka T, Ueno M, Shigekawa Y, Kawakami Y, Arihiro K, Ohdan H, Gotoh K, Ishikawa O, Ariizumi S, Yamamoto M, Yamada T, Chayama K, Kosuge T, Yamaue H, Kamatani N, Miyano S, Nakagama H, Nakamura Y, Tsunoda T, Shibata T, Nakagawa H: Whole-genome sequencing of liver cancers identifies etiological influences on mutation patterns and recurrent mutations in chromatin regulators. Nat Genet 2012;44:760-764.

$>5$ Huang J, Deng Q Wang Q Li KY, Dai JH, Li N, Zhu ZD, Zhou B, Liu XY, Liu RF, Fei QL, Chen H, Cai B, Zhou B, Xiao HS, Qin LX, Han ZG: Exome sequencing of hepatitis B virus-associated hepatocellular carcinoma. Nat Genet 2012;44:1117-1121.

-6 Kan Z, Zheng H, Liu X, Li S, Barber TD, Gong Z, Gao H, Hao K, Willard MD, Xu J, Hauptschein R, Rejto PA, Fernandez J, Wang G, Zhang Q Wang B, Chen R, Wang J, Lee NP, Zhou W, Lin Z, Peng Z, Yi K, Chen S, Li L, Fan X, Yang J, Ye R, Ju J, Wang K, Estrella H, Deng S, Wei P, Qiu M, Wulur IH, Liu J, Ehsani ME, Zhang C, Loboda A, Sung WK, Aggarwal A, Poon RT, Fan ST, Wang J, Hardwick J, Reinhard C, Dai H, Li Y, Luk JM, Mao $\mathrm{M}$ : Whole-genome sequencing identifies recurrent mutations in hepatocellular carcinoma. Genome Res 2013;23:1422-1433.

7 Totoki Y, Tatsuno K, Yamamoto S, Arai Y, Hosoda F, Ishikawa S, Tsutsumi S, Sonoda K, Totsuka H, Shirakihara T, Sakamoto H, Wang L, Ojima H, Shimada K, Kosuge T, Okusaka T, Kato K, Kusuda J, Yoshida T, Aburatani H, Shibata T: High-resolution characterization of a hepatocellular carcinoma genome. Nat Genet 2011;43:464469. 


\section{Cellular Physiology Cell Physiol Biochem 2018;47:2602-2612 \begin{tabular}{l|l} 
DOI: 10.1159/000491656 & $\begin{array}{l}\text { O 2018 The Author(s). Published by S. Karger AG, Basel } \\
\text { www.karger.com/cpb }\end{array}$ \\
\hline
\end{tabular}}

Dong et al.: CDK13 RNA Over-Editing in HCC

8 Benne R: RNA-editing in trypanosome mitochondria. Biochim Biophys Acta 1989;1007:131-139.

9 Bass BL: RNA editing by adenosine deaminases that act on RNA. Annu Rev Biochem 2002;71:817-846.

10 Maas S, Rich A, Nishikura K: A-to-I RNA editing: recent news and residual mysteries. J Biol Chem 2003;278:1391-1394.

11 Hu X, Wan S, Ou Y, Zhou B, Zhu J, Yi X, Guan Y, Jia W, Liu X, Wang Q, Qi Y, Yuan Q Huang W, Liao W, Wang Y, Zhang Q Xiao H, Chen X, Huang J: RNA over-editing of BLCAP contributes to hepatocarcinogenesis identified by whole-genome and transcriptome sequencing. Cancer Lett 2015;357:510-519.

$>12$ Chen L, Li Y, Lin CH, Chan TH, Chow RK, Song Y, Liu M, Yuan YF, Fu L, Kong KL, Qi L, Li Y, Zhang N, Tong AH, Kwong DL, Man K, Lo CM, Lok S, Tenen DG, Guan XY: Recoding RNA editing of AZIN1 predisposes to hepatocellular carcinoma. Nat Med 2013;19:209-216.

13 John D, Weirick T, Dimmeler S, Uchida S: RNAEditor: easy detection of RNA editing events and the introduction of editing islands. Brief Bioinform 2017;18:993-1001.

14 Xie C, Mao X, Huang J, Ding Y, Wu J, Dong S, Kong L, Gao G, Li CY, Wei L: KOBAS 2.0: a web server for annotation and identification of enriched pathways and diseases. Nucleic Acids Res 2011;39: 316-322.

15 Ramaswami G, Lin W, Piskol R, Tan MH, Davis C, Li JB: Accurate identification of human Alu and non-Alu RNA editing sites. Nat Methods 2012;9:579-581.

16 Wong SK, Sato S, Lazinski DW: Elevated activity of the large form of ADAR1 in vivo: very efficient RNA editing occurs in the cytoplasm. RNA 2003;9:586-598.

17 Kiran A, Baranov PV: DARNED: a DAtabase of RNa EDiting in humans. Bioinformatics 2010;26:1772-1776.

18 Porath HT, Carmi S, Levanon EY: A genome-wide map of hyper-edited RNA reveals numerous new sites. Nat Commun 2014;5:4726.

19 Cai ZX, Chen G, Zeng YY, Dong XQ, Lin MJ, Huang XH, Zhang D, Liu XL, Liu JF: Circulating tumor DNA profiling reveals clonal evolution and real-time disease progression in advanced hepatocellular carcinoma. Int J Cancer 2017;141:977-985.

20 Avesson L, Barry G: The emerging role of RNA and DNA editing in cancer. Biochim Biophys Acta 2014;1845:308-316.

-21 Chen CX, Cho DS, Wang Q, Lai F, Carter KC, Nishikura K: A third member of the RNA-specific adenosine deaminase gene family, ADAR3, contains both single- and double-stranded RNA binding domains. RNA 2000;6:755-767.

22 Greifenberg AK, Honig D, Pilarova K, Duster R, Bartholomeeusen K, Bosken CA, Anand K, Blazek D, Geyer M: Structural and Functional Analysis of the Cdk13/Cyclin K Complex. Cell Rep 2016;14:320-331.

23 Even Y, Escande ML, Fayet C, Geneviere AM: CDK13, a Kinase Involved in Pre-mRNA Splicing, Is a Component of the Perinucleolar Compartment. PLoS One 2016;11:e0149184.

24 Liang K, Gao X, Gilmore JM, Florens L, Washburn MP, Smith E, Shilatifard A: Characterization of human cyclin-dependent kinase 12 (CDK12) and CDK13 complexes in C-terminal domain phosphorylation, gene transcription, and RNA processing. Mol Cell Biol 2015;35:928-938.

25 Kim HE, Kim DG, Lee KJ, Son JG, Song MY, Park YM, Kim JJ, Cho SW, Chi SG, Cheong HS, Shin HD, Lee SW, Lee JK: Frequent amplification of CENPF, GMNN and CDK13 genes in hepatocellular carcinomas. PLoS One 2012;7:e43223.

-26 Sakurai M, Ueda H, Yano T, Okada S, Terajima H, Mitsuyama T, Toyoda A, Fujiyama A, Kawabata H, Suzuki T: A biochemical landscape of A-to-I RNA editing in the human brain transcriptome. Genome Res 2014;24:522-534.

27 Maas S, Godfried Sie CP, Stoev I, Dupuis DE, Latona J, Porman AM, Evans B, Rekawek P, Kluempers V, Mutter M, Gommans WM, Lopresti D: Genome-wide evaluation and discovery of vertebrate A-to-I RNA editing sites. Biochem Biophys Res Commun 2011;412:407-412.

28 Kim U, Wang Y, Sanford T, Zeng Y, Nishikura K: Molecular cloning of cDNA for double-stranded RNA adenosine deaminase, a candidate enzyme for nuclear RNA editing. Proc Natl Acad Sci U S A 1994;91:11457-11461.

29 Kang L, Liu X, Gong Z, Zheng H, Wang J, Li Y, Yang H, Hardwick J, Dai H, Poon RT, Lee NP, Mao M, Peng Z, Chen R: Genome-wide identification of RNA editing in hepatocellular carcinoma. Genomics 2015;105:7682.

-30 Chan TH, Lin CH, Qi L, Fei J, Li Y, Yong KJ, Liu M, Song Y, Chow RK, Ng VH, Yuan YF, Tenen DG, Guan XY, Chen L: A disrupted RNA editing balance mediated by ADARs (Adenosine DeAminases that act on RNA) in human hepatocellular carcinoma. Gut 2014;63:832-843. 